\title{
Acute Severe Autonomic Dysreflexia during Spinal Cord Intramedullary Tumor Resection
}

\author{
Guive Sharifi ${ }^{1}$, Elham Saedi ${ }^{2}$, Mohammad Ali Arami ${ }^{3 *}$ \\ ${ }^{1} \mathrm{MD}$, Associate Professor, Department of Neurosurgery, Loghman Hospital, Shahid Beheshti University of Medical Sciences, Tehran, Iran \\ ${ }^{2}$ MD, Neurologist, Department of Neurology, Amiralam Hospital, Tehran University of Medical Sciences, Tehran, Iran \\ ${ }^{3} \mathrm{MD}$, Consultant Neurologist, Department of Neurology, Milad Hospital, School of Medicine, Tehran University of Medical Sciences, Tehran, Iran \\ * Corresponding Author Address: Department of Neurology, Milad General Hospital, Tel: +98218230000, Fax: +98 218230001, \\ E-mail: arami_ma@yahoo.com
}

Article Type: Brief Report

Received: December 13, 2015, Last revised: December 15, 2015, Accepted: December 25, 2015

\section{Abstract}

Background \& Importance: Acute autonomic system impairment can be dangerous and life-threatening, and a clinician should be aware of its occurrence possibility. According to the anatomy of this system, spinal cord lesions could disturb its function. There are many reports of autonomic dysreflexia in spinal cord injuries especially spinal cord trauma.

Case Presentation: A 28 year-old man with an eight month history of gradual numbness in his extremities referred to our university hospital. Neurologic examination revealed mild spastic motor weakness in all limbs and increased deep tendon reflexes.

Conclusion: Acute and dangerous autonomic dysreflexia during spinal cord surgery is a rare condition that neurosurgeons and anesthesiologists should be ready for immediate diagnosis and treatment of this condition.

Keywords: Autonomic Dysreflexia; Intramedullary Tumor

Please cite this paper as: Sharifi G, Saedi E, Arami M.A. Acute Severe Autonomic Dysreflexia during Spinal Cord Intramedullary Tumor

Resection. Iran. J. Neurosurg. 2015;1(4):28-29

\section{Introduction}

Acute autonomic system impairment can be dangerous and lifethreatening, and a clinician should be aware of its occurrence possibility. According to the anatomy of this system, spinal cord lesions could disturb its function. There are many reports of autonomic dysreflexia in spinal cord injuries especially spinal cord trauma (1). Acute and dangerous autonomic dysreflexia during spinal cord surgery is a rare condition that neurosurgeons and anesthesiologists should be ready for immediate diagnosis and treatment of this condition.

\section{Case Presentation}

A 28 year old man with an eight month history of gradual numbness of his extremities referred to our university hospital. Neurologic examination revealed mild spastic motor weakness in all limbs and increased deep tendon reflexes. Plantar reflexes were extensor, and Hoffman's sign was found in both hands. There were no symptoms related to urethral or anal sphincters' functions.

Brain and cervical cord Magnetic Resonance Imaging (MRI) was performed to rule out the existence of structural lesions in these areas. Neuroimaging demonstrated severe enlargement of the cervical spinal cord at the level of C2-C6 with an ill-defined region of slightly high signal intensity at T1-weighted imaging. The lesion demonstrated a mild heterogeneous enhancement after contrast injection. Myelography revealed blocking of the perimedullary subarachnoid spaceas (Figures 1,2). The brain imaging findings were normal.

\section{Surgical Procedure}

Laminectomy was performed from $\mathrm{Cl}$ to $\mathrm{C} 5$. After incision of the dura, an enlarged spinal cord was exposed. Myelotomy was done under sensory and motor tracts monitoring by our intraoperative neuromonitoring team. The tumor was resected by ultrasonic aspirator, and attempts were made to perform total resection of the tumor. During the first attempt to do dissection of tumor, the patient's heart rate dropped sharply and heartbeat stopped within a few seconds. The patient's heart rate and blood pressure returned to normal level, after a few seconds of stopping surgery and spinal manipulation and injecting atropine. The surgery continued with the agreement of anesthetic team, but the patient's blood pressure suddenly went up from $120 / 80$ to $230 / 130 \mathrm{mmHg}$ during the second attempt to remove the tumor. Therefore, the surgery was stopped for a few minutes, and the patient's blood pressure was controlled with drugs, and cardiac monitoring continued. After stabilizing the patient's hemodynamic condition, the surgery resumed with great caution. Fortunately, it continued without hemodynamic compromise.

In the final minutes of surgery, MEP amplitude on the right side significantly decreased. Homeostasis was achieved after near total resection of the tumor, and the dura was repaired. Postoperative paresis with strength of $3 / 5$ occurred in the right leg that improved to $4 / 5$ after one week, and he had very mild weakness in his leg after 3 months. 


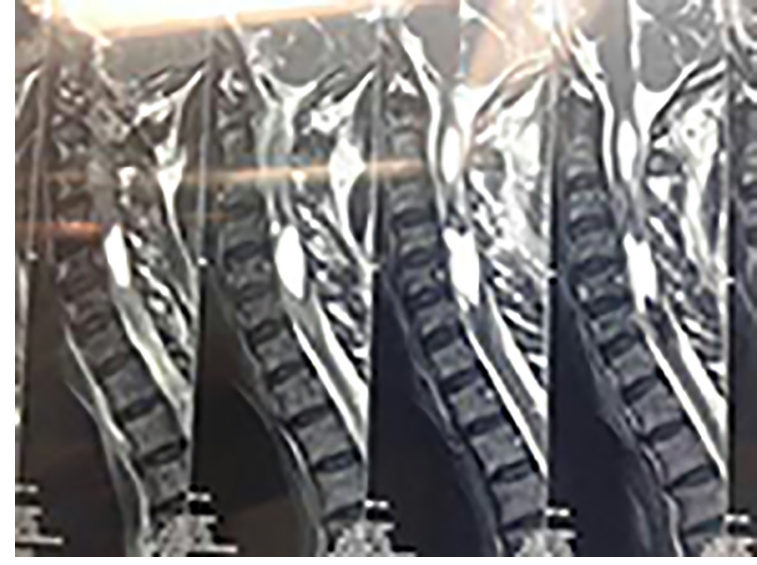

Figure 1. Cervical T1 MRI

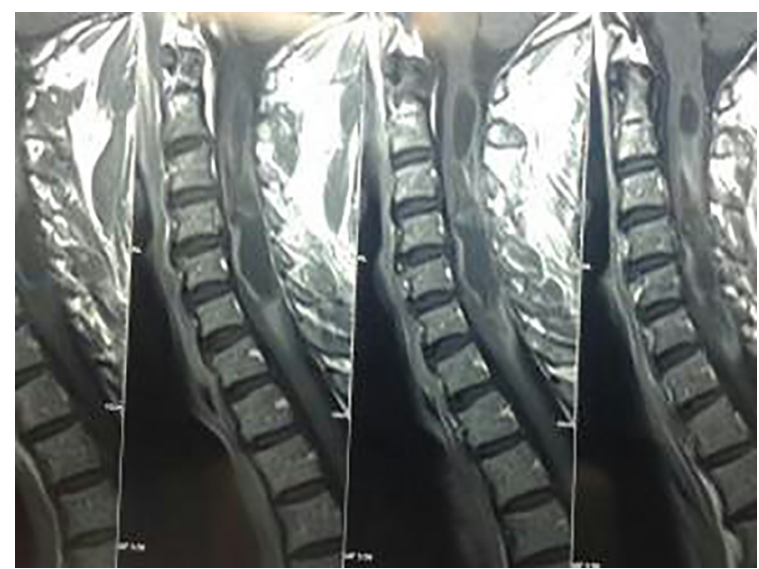

Figure 2. Cervical T2 MRI

\section{Discussion}

Autonomic dysreflexia has long been recognized in the spinal cord injury. Diagnosis and treatment of autonomic dysreflexia is important in patients with spinal cord injury, so the healthcare team needs to have the skills to deal with these problems.

There are numerous articles and reports in the medical literature that explained the mechanisms of these disorders. Autonomic dysreflexia can be asymptomatic, but it can present with various signs and symptoms including fluctuating blood pressure, heart rate disturbances, anxiety, blurred vision, headache, flushing, and sweating above the level of injury. Autonomic bladder and gastrointestinal problems could result in important complications. However, acute autonomic dysreflexia is of special importance. In serious cases, the severe hypertension crisis can result in loss of consciousness due to cerebral hemorrhage, seizures, and pulmonary edema (2). Cardiovascular function disturbances are potentially lifethreatening and demanding more attention (3).

Although autonomic dysreflexia is often seen with spinal cord trauma, nontraumatic lesions such as spinal cord tumors, spinal cord infarction and multiple sclerosis plaques can cause autonomic dysreflexia and its complications (4). On the other hand, it may also be seen during surgery. Surgeon and anesthesiologist must remain vigilant, as the case may be quite severe and without warning.

According to reports, episodic hypertension could develop in $50-90 \%$ of people who suffered tetraplegia or high thoracic cord lesions (5). This reflex could be seen in patients with complete or incomplete injury to the T6 or higher levels of cord (6), although autonomic dysreflexia has been reported with injuries to T8 level of spinal cord. (7). It sould be noted that sometimes the spinal cord injury may not be suspected without a complete history because incomplete spinal cord injury may allow the patient to walk. This condition could be caused by spinal reflex mechanisms because they remain intact despite the patient's spinal cord injury (8).

\section{References}

1. Craig A. Bauman, James D. Milligan, F. Joseph Lee, John J. Riva. Autonomic dysreflexia in spinal cord injury patients: an overview. J Can Chiropr Assoc 2012:56(4):247-250

2. Amber Eker, Pembe Hare Yigitoglu, H. Ilker Ipekdal, Aliye Tosun. Acute Onset of Intracerebral Hemorrhage due to Autonomic Dysreflexia. J Korean Neurosurg Soc. 2014;55(5):277-279.

3. Sven Magnus Hector, Tor Biering-Sørensen, Andrei Krassioukov, Fin Biering-Sørensen. Cardiac arrhythmias associated with spinal cord injury. The Journal of Spinal Cord Medicine 2013;36(6):591-599

4. Bateman AM, Goldish GD. Autonomic dysreflexia in multiple sclerosis. J Spinsl Cord Med, 2002;25(1):40-42.

5. Vaidyanathan S. Autonomic dysreflexia in a tetraplegic patient due to a blocked urethral catheter: spinal cord injury patients with lesions above T-6 require prompt treatment of an obstructed urinary catheter to prevent lifethreatening complications of autonomic dysreflexia. International J Emerg Med. 2012;5(6):1-5.

6. Middleton J. Management of spinal cord injury in general practice - Part 1 . Australian Family Physician. 2008;37(4):229-34.

7. Moeller B. Autonomic dysreflexia in injuries below the sixth thoracic segment. JAMA. 1973;224(9):1295.

8. Blackmer J. Rehabilitation medicine: 1. Autonomic dysreflexia. CMAJ 2003;169(9):931-35. 